\title{
Effects of Temperature dependent viscosity on Penetrative Convection in a Fluid Layer Bounded by Slabs of Finite Thermal Conductivity and Finite Thickness
}

\author{
Gangadharaiah Y.H. ${ }^{1^{*}}$, Ananda $\mathbf{K}^{2}$ \\ ${ }^{1}$ Department of Mathematics, Sir M. Visvesvaraya Institute of Technology, Bangalore-562157, India \\ ${ }^{2}$ Department of Mathematics, New Horizon College of Engineering, Bangalore-560 103, India
}

Available online at: www.isroset.org

Received: 07/Sept/2018, Accepted:23/Sept/2018, Online: 31/Oct/2018

\begin{abstract}
The onset of penetrative convection in a variable-viscosity flow bounded by slabs of finite thermal conductivity and finite thickness has been investigated by means of linear stability analysis. The relationship between the viscosity and the temperature is assumed of exponential type. The asymptotic solutions of the long wavelength, for small values of the conductivity and thickness of the solid, are achieved. The values of critical Rayleigh numbers for different values of thermal conductivity ratio and viscosity parameters and, consequently the critical Rayleigh numbers at which the onset of convection starts, are computed analytically. The effects of various parameters (namely, viscosity parameter, thermal conductivity ratio , depth ratio and the presence of internal heat source strength on the onset of stationary convection) are computed analytically and depicted graphically. It is observed that both stabilizing and destabilizing factors can be enhanced because of the presence of a heat source, thermal conductivity ratio, depth ratio, and variable viscosity .As a result, it is possible so that to postpone (or advance) significantly the onset of motion.
\end{abstract}

Keywords: variable viscosity: internal heat source: thermal conductivity: Stability.

\section{INTRODUCTION}

It is well known that the temperature dependence of fluid properties in flows with heat transfer can change the flow behaviour: especially its stability characteristics. For most of the realistic fluids, the viscosity shows a rather pronounced variation with respect to temperature, as viscosity is more sensitive to temperature than heat capacity and thermal conductivity. Rossby [1] computed the values of viscosity and thermal conductivity for water between 20 and $25^{\circ} \mathrm{C}$ and observed that the variation in kinematic viscosity is approximately $10 \%$ between 20 to $25^{\circ} \mathrm{C}$, whereas the thermal conductivity of water varies only by $1.5 \%$. Torrance and Turcotte [2] noted that the viscosity of fluids decreases with increasing temperature, whereas a reverse trend is observed in gases. In recent years, many authors investigated the influence of temperature dependent viscosity in the problems of Rayleigh-Benard convection. Booker [3] has studied Rayleigh-Benard convection with strongly temperature-dependent viscosity. Booker and Stengel [4] have shown that there is a decrease in convective heat transport due to the increase in the critical Rayleigh number with variable viscosity. Early studies related to the convection in fluids with temperature dependent viscosity are those of Palm [5], in which the linear dependence of viscosity with the temperature is adopted, and of Stengel et al. [6], in which they considered viscosity depending exponentially on temperature.

Jenkins [7] considered the general dependence of viscosity on temperature and studied both linear and exponential dependence of viscosity, and he concluded that the linear dependence is realistic for fluids with small values of viscosity, whereas exponential dependence is more realistic for fluids with high viscosity. Recently, Dhiman and Kumar [8] investigated the onset of stationary Rayleigh-Benard convection with variable viscosity for all combinations of rigid and dynamically free boundaries using the Galerkin method and concluded that the positive values of the temperature-dependent viscosity parameter have a stabilizing effect on the onset of stationary convection, whereas negative values have a destabilizing effect. Many other authors, who include Selak and Lebon [9],Nield [10]and Straughan [11], have also investigated the onset of convection for the ordinary fluids with strongly temperature-dependent viscosity. Palm [5] discussed the effect of a variable viscosity on the Benard convection problem and, from the observed results, he explained theoretically that the cells in steady convection 
approach a hexagonal form, and the occurrence of ascent or descent in the middle of the cells depends on how the viscosity varies on the Rayleigh's result. Stengel et al. [6] compared Palm's results [5] with those arising from the selection of an exponential viscosity variation law. Further, this problem was also studied by Busse and Frick [12] for low-viscosity fluids with a linear dependence of the viscosity on temperature. According to Solomatov [13], when the viscosity ratio exceeds 3000, a stagnant lid regime occurs, where a thick cold boundary layer develops at the top plate. Such regime was observed for $R_{a}$, up to 108 by Davaille and Jaupart [14].

We examine the linear stability of variable-viscosity flow between slabs of finite thermal conductivity and finite thickness due to an applied pressure gradient in the presence of an applied vertical temperature gradient and uniform internal heat source. We believe that this problem is paradigmatic to the very general problem involving the interaction between a nonuniform applied temperature gradient and a variable-viscosity flow. The results are relevant to current industrial applications involving chemical vapour deposition or the cooling of electronic equipment; see e.g. ([15-19]).

The objective of the present study is to investigate the influences of the varying viscosity with internal heat generation between the solid plates of finite thickness and of finite conductivity. The linear stability theory is applied and the resulting eigenvalue problem is solved by analytically using regular perturbation technique. The critical Rayleigh number $R_{c}$, which depend on related physical parameters, are investigated. This paper is organized as follows. First, mathematical formulation of the problem and the linear stability theory is used in order to predict the critical Rayleigh number $R_{c}$, for the onset of convection is presented in Sec. 2. Finally, the results from the analytical computations are discussed and conclusions are drawn in Sec. 4 and Sec. 5.

\section{PHYSICAL CONFIGURATION AND EIGENVALUE PROBLEM}

The system under investigation is shown schematically in Fig. 1. An infinite horizontal fluid layer of thickness $d$, confined between two identical homogeneous, isotropic solid plates of thickness $d_{s}$, thermal diffusivity $D_{s}$ and thermal conductivity $k_{s}$, above and below the fluid. A uniform internal heat generation per unit volume $q$, is applied on the layer.

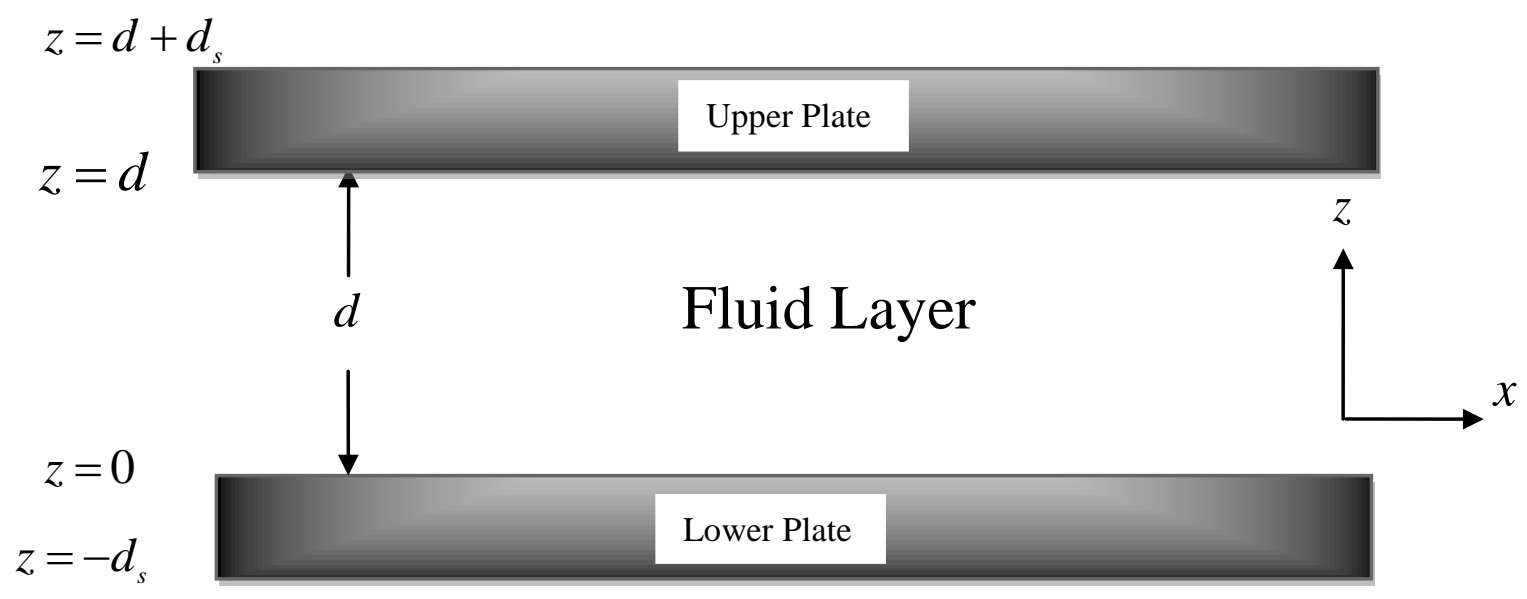

Figure 1: Physical configuration

The governing equations for the fluid and the solid layers are :

Fluid layer $(0 \leq z \leq d)$ :

$\nabla \cdot \vec{V}=0$ 
$\rho_{0}\left(\frac{\partial \vec{V}}{\partial t}+(\vec{V} \cdot \nabla) \vec{V}\right)=-\nabla p+\rho_{0} \vec{g}\left[1-\alpha\left(T-T_{0}\right)\right]+2 \nabla \cdot\left[\mu\left(\nabla \cdot \vec{V}+\nabla \cdot \vec{V}^{T}\right)\right]$

$\frac{\partial T}{\partial t}+(\vec{V} \cdot \nabla) T=k \nabla^{2} T+q$

Solid layer $\left(-d_{s} \leq z \leq 0\right.$ and $\left.d \leq z \leq d+d_{s}\right)$ :

$\frac{\partial T_{s}}{\partial t}=D_{s} \nabla^{2} T_{s}$

where

$\mu=\mu_{0} \exp \left[-A\left(T-T_{0}\right)\right]$

and $\mu_{0}$ is the dynamic viscosity corresponding to a temperature equal to the mean of temperature at the boundaries, $\vec{V}=(u, v, w)$ is the velocity vector, $p$ is the pressure, $T$ is the temperature, $g$ is the acceleration of gravity, $\rho_{0}$ is the reference fluid density.

The basic state is quiescent and is of the form

$$
(u, v, w, p, T)=\left[0,0, W_{0}, p_{b}(z), T_{b}(z)\right]
$$

The basic steady state is assumed to be quiescent and temperature distributions are found to be

$$
T_{b}(z)=T_{0}-\left[\left(\frac{\left(T_{0}-T_{u}\right)}{d}-\frac{q d}{2 \kappa}\right) z+\frac{q}{2 \kappa} z^{2}\right]
$$

The governing equations are nondimensionalized by scaling the length with $d$, time with $d^{2} / \kappa$, velocity with $\kappa / d$, and temperature in the fluid layer $T_{0}-T_{u}$. In order to investigate the stability of the basic solution, infinitesimal disturbances are introduced in the form

$$
\vec{V}=\vec{V}^{\prime}, \quad T=T_{b}+T^{\prime}, \quad p=p_{b}+p^{\prime}, \quad \rho=\rho_{b}+\rho^{\prime}, \quad \mu=\mu_{b}+\mu^{\prime}
$$

Using these scales, Eqs. 2-4 can be transformed to the following dimensionless form:

$$
\begin{aligned}
& \frac{1}{p r} \frac{\partial}{\partial t} \nabla^{2} w=\tilde{f} \nabla^{4} w+2 \frac{\partial \tilde{f}}{\partial z} \nabla^{2} \frac{\partial w}{\partial z}+\frac{\partial^{2} \tilde{f}}{\partial z^{2}}\left(\nabla^{2} w-2 \nabla_{h}^{2} w\right)+R \nabla_{h}^{2} T \\
& \left(\frac{\partial}{\partial t}-\nabla^{2}\right) T=w[1-Q(1-2 z)] \\
& \frac{\partial \theta_{s}}{\partial t}=\frac{D_{s}}{D_{f}} \nabla^{2} \theta_{s}
\end{aligned}
$$


where $R=\alpha g\left(T_{0}-T_{u}\right) d^{3} / v \kappa$ is the Rayleigh number, $Q=q d^{2} / 2 \kappa\left(T_{0}-T_{u}\right)$ is the dimensionless heat source strength and $\nabla^{2}=\nabla_{h}^{2}+\partial^{2} / \partial z^{2}$ is the Laplacian operator with $\nabla_{h}^{2}=\partial^{2} / \partial x^{2}+\partial^{2} / \partial y^{2}$. The function $\tilde{f}$ representing the temperature dependence of viscosity, is defined as

$$
\tilde{f}=\exp \left[B\left(z-\frac{1}{2}\right)\right], \quad B=\left(\frac{v_{\max }}{v_{\min }}\right) .
$$

We assume that the perturbations $w, T$ and $\theta_{s}$ have the forms :

$$
\left(w, T, \theta_{s}\right)=\left[W(z), \Theta(z), \theta_{s}(z)\right] \exp [i(l x+m y)]
$$

and substituting them in Eqs. $(9)-(11)$ (with $\partial / \partial t=0)$, we obtain the following ordinary differential equations

$$
\begin{aligned}
& \tilde{f}\left(D^{2}-a^{2}\right)^{2} W+2 D \tilde{f}\left(D^{2}-a^{2}\right) D W+D^{2} \tilde{f}\left(D^{2}+a^{2}\right) W=R a^{2} \Theta \\
& \left(D^{2}-a^{2}\right) \Theta=-W[1-Q(1-2 z)] \\
& \left(D^{2}-a^{2}\right) \Theta_{s}=0 .
\end{aligned}
$$

The boundary conditions are:

$$
\begin{array}{lccc}
D^{2} w=0 & \text { at } & z=1 . \\
w=0, & D w=0, & \text { at } & z=0,1 \\
\theta=\theta_{s}, & D \theta=k_{r} D \theta_{s} & \text { at } & z=0,1 \\
D \theta_{s}=0 & \text { at } & z=-d_{r}, 1+d_{r} .
\end{array}
$$

Here, $d_{r}=d_{s} / d$ is the ratio of the solid plate thickness to the liquid layer thickness and $k_{r}=k_{s} / k_{f}$ is the ratio of the thermal conductivity of the solid plate to that of the fluid layer. Solving Eq. (16) for the solid layer, together with the boundary conditions (19) and (20), the thermal boundary condition at the solid-fluid interface becomes

$$
\begin{aligned}
& D \Theta=k_{r} a \tanh \left(a d_{r}\right) \Theta . \quad \text { at } z=0 \\
& D \Theta=k_{r} a \tanh \left(a\left(2+d_{r}\right)\right) \Theta . \quad \text { at } z=1
\end{aligned}
$$

\section{LONG WAVELENGTH ASYMPTOTIC ANALYSIS}

The solution of the governing Eqs. 14-16 and boundary conditions Eqs. 17-20 is obtained using a regular perturbation technique with wave number $a$ as a perturbation parameter.

For studying the validity of the small wave number analysis, the variables $W$ and $\Theta$ are expressed in terms of the small wave number $a$, 
$(W, \Theta)=\sum_{i=0}^{N}\left(a^{2}\right)^{i}\left(W_{i}, \Theta_{i}\right)$

Substitution of Eqs. (22) into Eqs. (14) - (16) and the boundary conditions (17) - (20), and collecting the terms of zeroth order, we obtain

$\tilde{f} D^{4} W_{0}+2 D \tilde{f} D^{3} W_{0}+D^{2} \tilde{f} D^{2} W_{0}=0$

$D^{2} \theta_{0}=-[1-Q(1-2 z)] W_{0}$

The boundary conditions are

$w_{0}(1)=D^{2} w_{0}(1)=D \theta_{0}(1)=0$

$w_{0}(0)=D w_{0}(0)=D \theta_{0}(0)=0$

Then solutions to above equations are

$W_{0}=0$ and $\Theta_{0}=1$

First- order equations are

$$
D^{4} W_{1}+2 B D^{3} W_{1}+B^{2} D^{2} W_{1}=R \operatorname{Exp}[-B(z-1 / 2)]
$$

$D^{2} \Theta_{1}=1-[1-Q(1-2 z)] W_{1}$.

The boundary conditions are

$D^{2} w_{1}(1)=0$

$D \theta_{1}(0)=k_{r} a \tanh \left(a d_{r}\right) \Theta_{0}(0)$

$$
D \theta_{1}(1)=k_{r} a \tanh \left(a\left(2+d_{r}\right)\right) \Theta_{0}(1) .
$$

The general solution of (30) is

$$
W_{1}=R\left[C_{1}+C_{2} z+C_{3} e^{-B z}+C_{4} z e^{-B z}+\frac{z^{2}}{2 B^{2}} e^{-B(z-1 / 2)}\right]
$$

where

$$
\begin{array}{ll}
C_{1}=-\frac{e^{B / 2}}{2 B^{2}}\left(\frac{e^{B}-B-1}{1-2 e^{B}+e^{2 B}-B^{2} e^{B}}\right), C_{2}=\frac{e^{B / 2}}{2 B^{2}}\left(\frac{2-2 e^{B}+B+B e^{B}}{e^{2 B}+2 e^{B}-B^{2} e^{B}-1}\right) \\
C_{3}=\frac{e^{B / 2}}{2 c^{2}}\left(\frac{e^{B}-B-1}{1+e^{2 B}-2 e^{B}-B^{2} e^{B}}\right), \quad C_{4}=\frac{e^{B / 2}}{2 B^{2}}\left(\frac{2-2 e^{B}+2 B+B^{2}}{2 e^{B}-e^{2 B}+B^{2} e^{B}-1}\right) .
\end{array}
$$

The differential Equation (30) involving $D^{2} \Theta_{1}$ provide the solvability requirement which is given by 
$\int_{0}^{1}[1-Q(1-2 z)] W_{1} d z=1-k_{r}\left(2+d_{r}\right)$

The expressions for $W_{1}$ is back substituted into Eq. (35) and integrated to yield an expression for the critical Rayleigh number $R_{c}$, which is given by

$R_{c}=\frac{12 B^{6} e^{\frac{2 B}{2}}\left(1-\left(2+d_{r}\right) k_{r}\right)\left(2+B^{2}-2 \cosh B\right)}{C_{1}+C_{2}\left(\frac{1}{2}+\frac{Q}{6}\right)+C_{3}\left(e^{B}\left(B_{r}-Q\right)-1\right)+C_{4}\left(e^{B}-1-\left(4 Q e^{B}-1\right)\right)+L_{1}}$

where

$L_{1}=\frac{1}{2 B^{3}}\left[(2 B-2) e^{B / 2}+2 e^{-B / 2}+N s\left((2-2 B) e^{-B / 2}-2 e^{B / 2}\right)\right]$.

\section{RESULTS AND DISCUSSION}

Thermal convective motion of fluid with internal heat generation and variable viscosity affected by walls of finite thickness and of finite conductivity were investigated by the linear analysis. The variable viscosity was assumed of exponential type. The resulting eigen value problem is solved analytically using a regular perturbation technique with wave number $a$ as a perturbation parameter. The marginal stability of the system considered in this investigation is given by equation (36). We can check this formula against known results for the following limiting case:

In the limit $Q \rightarrow 0$ and $B \rightarrow 0$, equation (36) is simplified to the following result, which is the case of a constant-viscosity and absence of internal heating in fluid layer between a solid walls of finite thickness and of finite conductivity,

$R_{c}=720\left(1-\left(2+d_{r}\right) k_{r}\right)$.

As $k_{r}=0$ or $d_{r}=0$, equation (37) can be reduced much further to the result $R_{c} \rightarrow 720$ which is the known exact value (Nield [20]).

Figure 2 depicts the perturbed vertical velocity profiles for different values $B$ for $Q=0$ and $k_{r}=0=d_{r}$. It shows that the appearance of newly formed sub layer, which first occurs at the maximum $R_{c}$ with associated viscosity parameter $B$, continues to manifest itself after then, becoming dominant at the critical state. As viscosity parameter is further increased, the viscously suppressive effects of main fluid flow above shorten the depth of sub layer and $R_{c}$ then decreases with viscosity parameter $B$. For the larger values of viscosity parameter $B$ the velocity profile vanishes at the lower part of fluid layer. Figure 3 shows the evolution of the maximum (minimum) value of vertical velocity, $W_{\max }\left(W_{\min }\right)$, predicted by the analytical solution of the full governing equations, as a function of time for different values of heat source strength $Q$ for viscosity parameter $B=3$. It is observed that the convection is closer to upper boundary with an increase of heat source strength $Q$. To gain physical insight into the onset of the convection, we illustrate the velocity profile for different cases for $Q, k_{r}, d_{r}$ and $B$. Similarly, from Figs.4,5,6 and 7, we observed that with increasing $Q, k_{r}, d_{r}$ and $B$ and the points where the maximum value of $W(z)$ takes place is near the bottom of the layer where the fluid is less viscous.

Figure 8 depicts the influence of the differential heat source strength $(Q)$ on the marginal stability curve, namely the critical Rayleigh number $R_{c}$ versus the viscosity parameter $B$ at the onset of motion, for $k_{r}=0=d_{r}$, it is observed that in the absence of internal heating $(Q=0)$ the critical Rayleigh number $R_{c}$ increases initially, with $B$, reaches maximum and then decreases with further increase in the value of $B$ and thus three regions are distinguished as observed in the case of 
isothermal boundary (see Stengel et al. [6]) $R_{c}$ increases only negligibly with $B$ for small values of $B$; increases significantly for $B$ up to about 8 or 9 , at which maximum values $R_{c}$ are reached; rapidly decreasing trends are found for values of $B$ above 9.Furthur increasing the internal heat source, loss of stability occurs into a time dependent motion, as also indicated by Fig. 8 .

Figures 9 and 10 reveals the effect $B$ on the values of $R^{c}$ for various values of thermal conductivity ratio $k_{r}$ and depth ratio $d_{r}$ with $Q=0$ and $Q=2$. It is clear from these figures that $R_{c}$ increases only negligibly with $B$ for small values of $B$; increases significantly for $B$ up to about 8 or 9 , at which maximum values $R_{c}$ are reached; rapidly decreasing trends are found for values of $B$ above 9. Further increasing thermal conductivity ratio $\left(k_{r}\right)$, depth ratio $\left(d_{r}\right)$ and internal heat source strength $(Q)$, system become more unstable. Figure 11 shows the variation of critical Rayleigh number $R_{c}$ with thermal conductivity ratio $k_{r}$ for different values depth ratio $d_{r}$ with $B=0=Q$ and $B=5, Q=2$. It is clear from the figure that $R_{c}$ decreases for both cases, where the influence of the conductivity ratio $k_{r}$ is completely negligible. Further increasing $B, d_{r}$ and $Q$ system become more unstable.

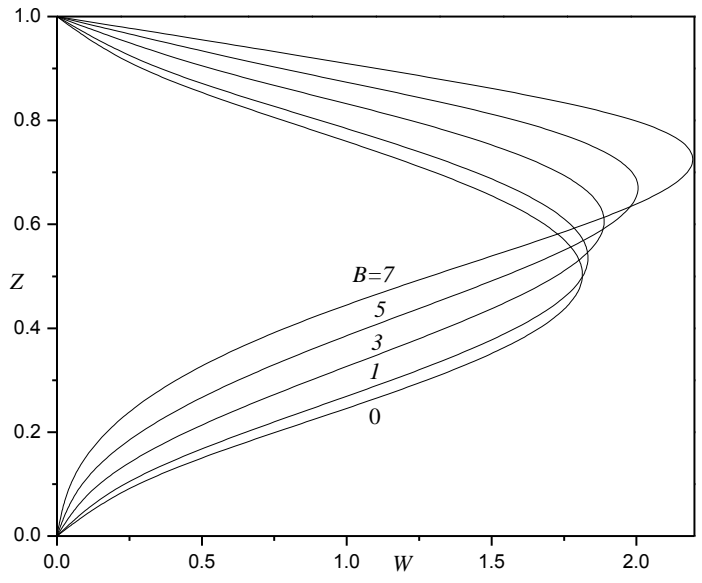

Figure 2: Vertical velocity profile for different values of $B$ with $N s=0$ and $k_{r}=0=d_{r}$

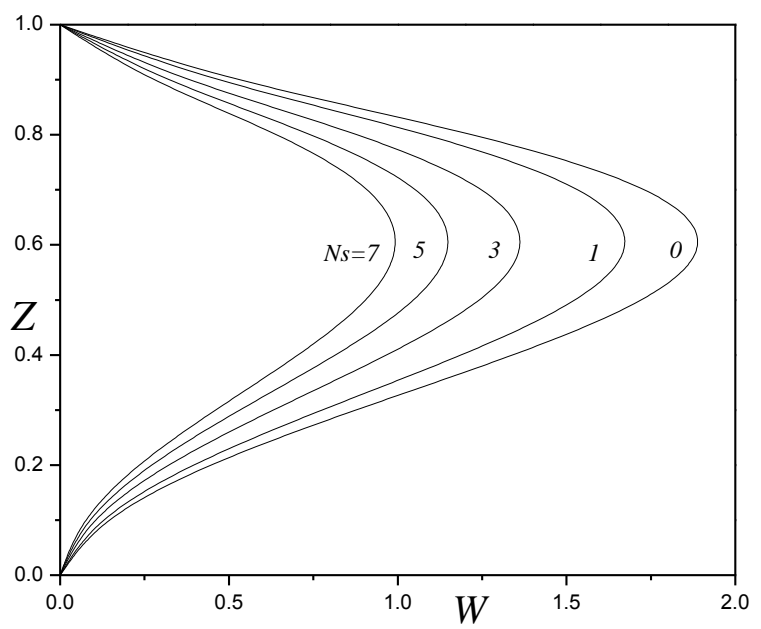

Figure 3: Vertical velocity profile for different values of $N s$ with $B=3$

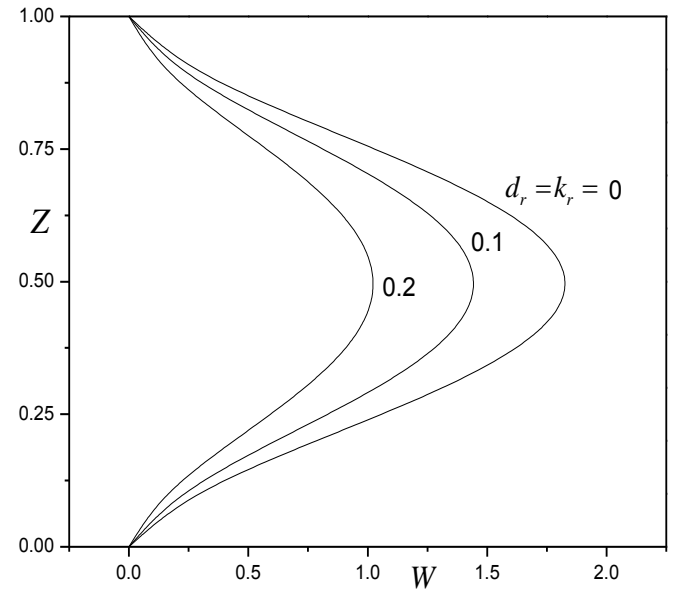

Figure 4: Vertical velocity profile for different values of $K_{r}, d_{r}$ with $B=0=Q$.

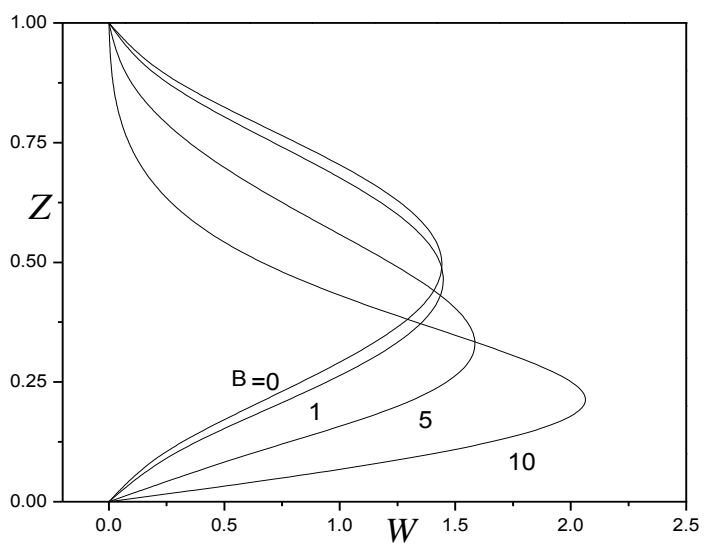

Figure 5: Vertical velocity profile for different values of $B$ with $Q=0, K_{r}=d_{r}=0.1$. 


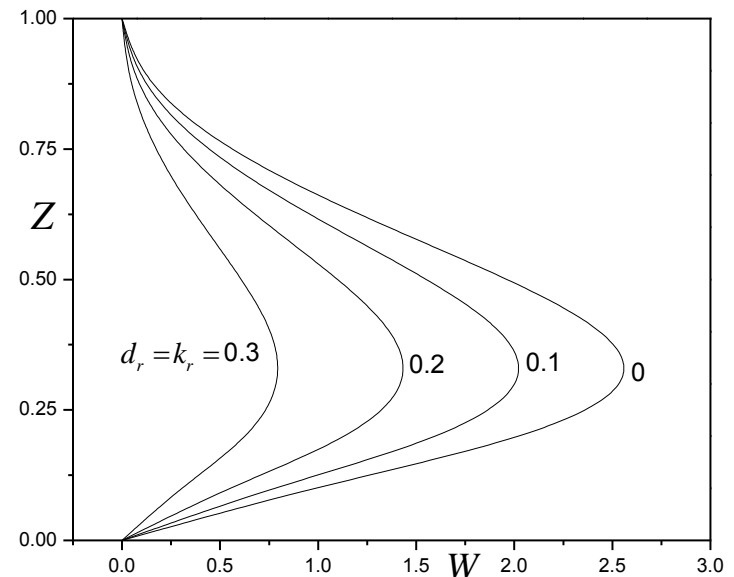

Figure 6: Vertical velocity profile for different values of $K_{r}, d_{r}$ with $Q=3, B=5$.

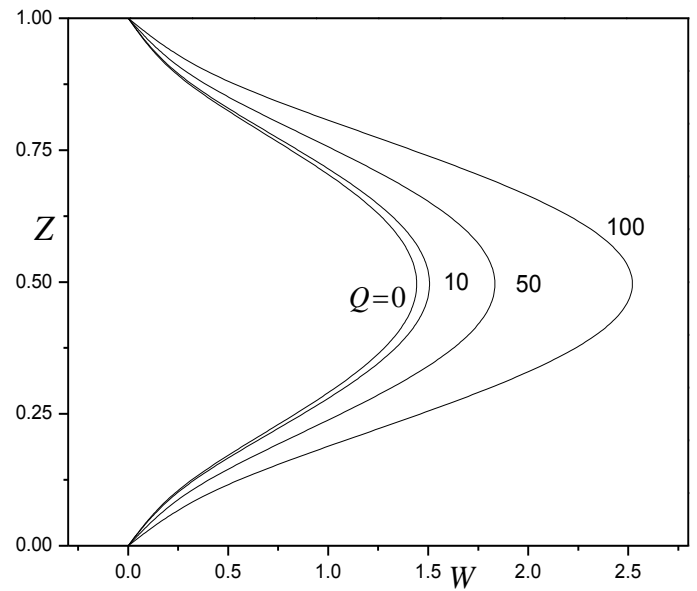

Figure 7: Vertical velocity profile for different values of $Q$ with $B=0, K_{r}=d_{r}=0.1$.

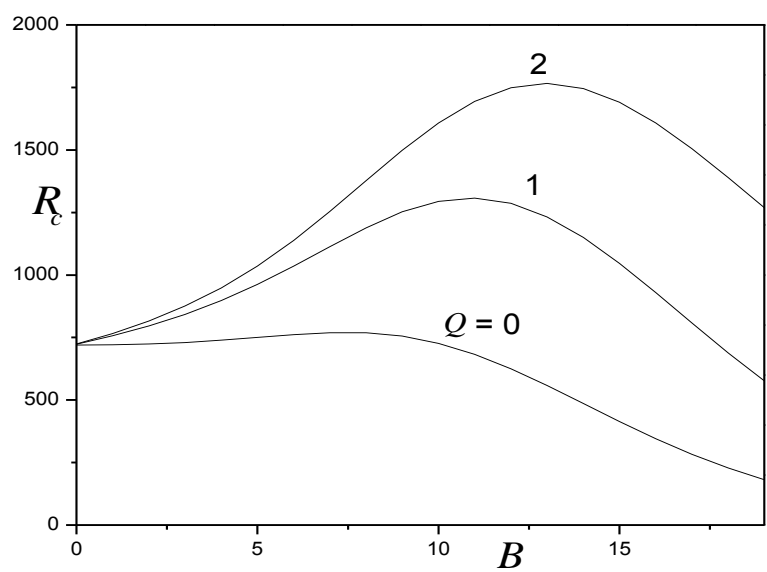

Figure 8: Influence of variable viscosity parameter $B$ for Critical for different values of $Q$ with $K_{r}=d_{r}=0$ on thermal convection.

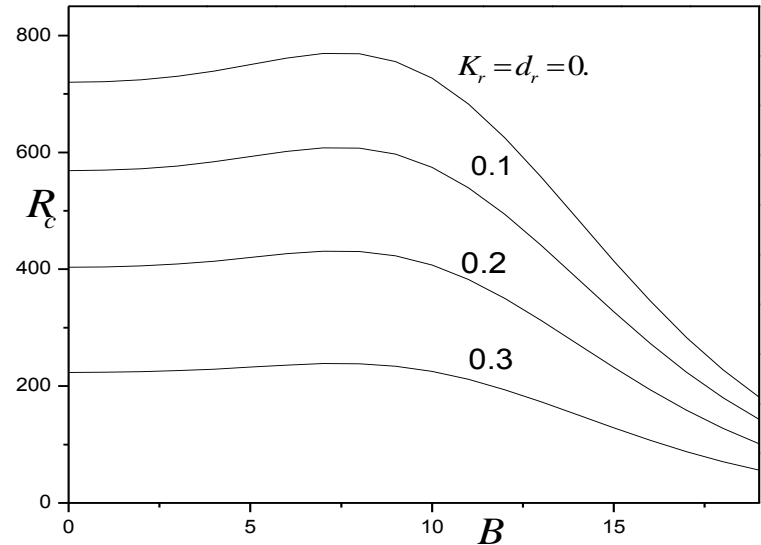

Figure 9: Influence of variable viscosity parameter $B$ for different values of $K_{r}$ and $d_{r}$ with $Q=0$ on thermal convection.

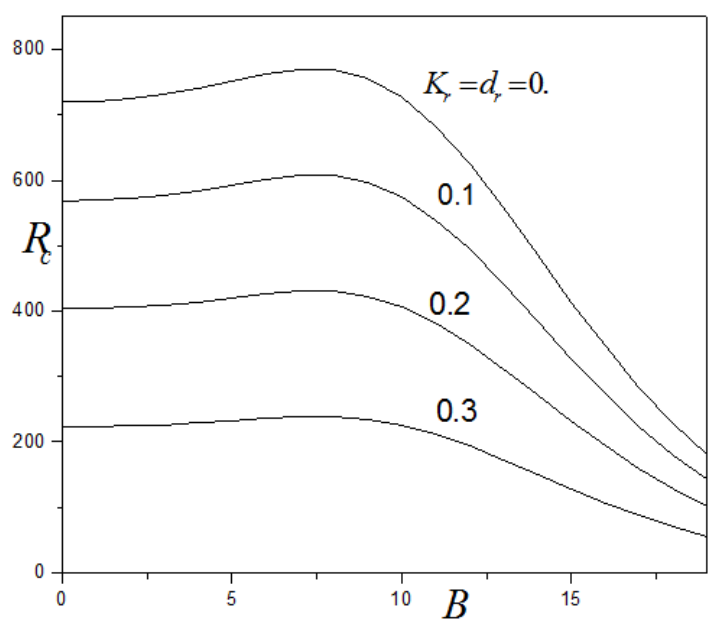

Figure 9: Influence of variable viscosity parameter $B$ for different values of $K_{r}$ and $d_{r}$ with $Q=0$ on thermal convection.

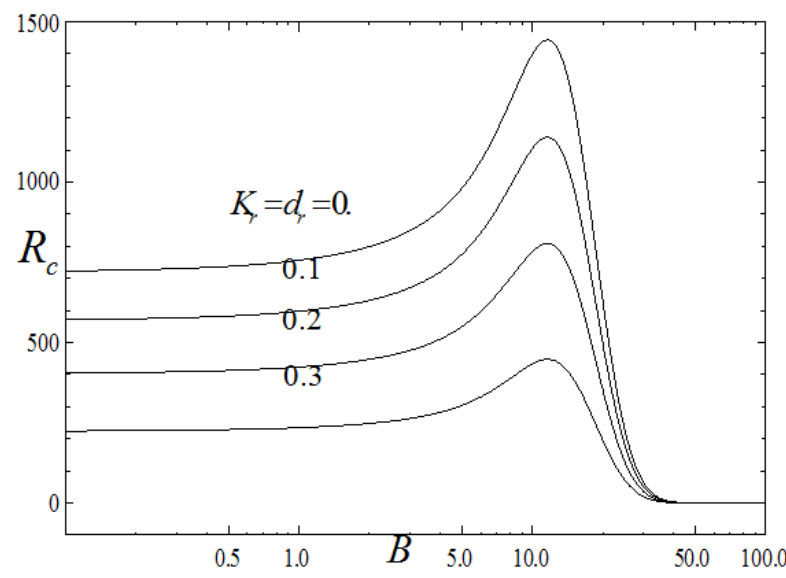

Figure 10: Influence of variable viscosity parameter $B$ for different values of $K_{r}$ and $d_{r}$ with $Q=2$ on thermal convection. 


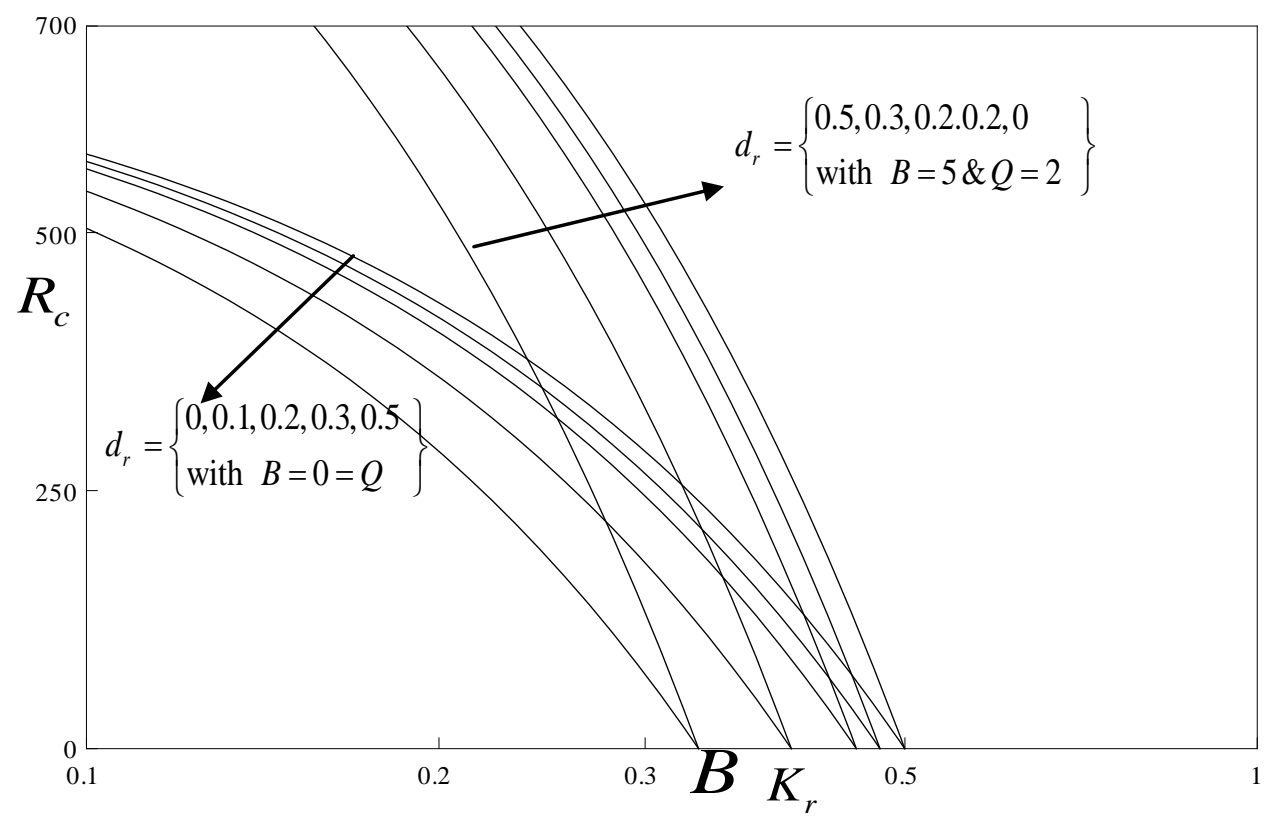

Figure 11: Influence of thermal conductivity ratio $K_{r}$ for different values of depth ratio $d_{r}$ on thermal convection.

\section{CONCLUSIONS}

The onset of penetrative convection via internal heating in a fluid layer bounded by solid plates is studied with an exponential viscosity variation. The asymptotic analysis of the long wavelength is performed and the results are compared with those for the case of constant-viscosity fluid. In the absence of internal heating $(Q=0)$, the $R_{c}$ increases only negligibly with viscosity parameter $B$ for small values of viscosity parameter $B$; increases significantly for $B$ up to about 8 or 9 , at which maximum values $R_{c}$ are reached; rapidly decreasing trends are found for values of $B$ above 9. Additionally, the characteristics of stability of the system are strongly dependent on the viscosity parameter $B$. A larger thermal conductivity ratio $k_{r}$ and depth ratio $d_{r}$ is destabilizing, the critical Rayleigh number $R_{c}$ decreases with increasing thermal conductivity ratio $k_{r}$ and depth ratio $d_{r}$ because an increase in the thermal conductivity ratio $k_{r}$ results in a destabilizing state, since thermal disturbances are easily dissipated deep into the solid layer, and the critical Rayleigh number $R_{c}$ decreases.

\section{REFERENCES}

[1] Rossby, H. T., “A Study of Bénard Convection with and Without Rotation,” Journal of Fluid Mechanics, Vol. 36,pp. 309-335,1969.

[2]. Torrance, K. E., and Turcotte, D. L., "Thermal Convection with Large ViscosityVariations,” Journal of Fluid Mechanics, Vol. 47, pp. 113-125, 1979.

[3]. Booker, J. R., “Thermal Convection with Strongly Temperature DependentViscosity,” Journal of Fluid Mechanics, Vol. 76, pp. 741-754, 1976.

[4]. Booker, J. R., and Stengel, K. C., "Further Thoughts on Convective Heat Transport in a Variable Viscosity Fluid," Journal of Fluid Mechanics, Vol. 86, pp. 289-291, 1978.

[5] Palm, E., "On the Tendency Towards Hexagonal Cells in Steady Convection,” Journal of Fluid Mechanics,Vol. 8, pp. 183-192, 1960.

[6] Stengel, K. C., Oliver, D. S., Booker, J. R., "onset of convection in a variable viscosity fluid", J. Fluid Mech.Vol. 120,pp. 411-431, 1982.

[7] Jenkins, D. R., "Rolls Versus Squares in Thermal Convection of Fluids with Temperature Dependent Viscosity," Journal of Fluid Mechanics, Vol. 178, pp. 491-506, 1987.

[8] Dhiman, J. S., and Kumar, V., "On Stability Analysis of Rayleigh Bénard Convection with Temperature Dependent Viscosity for General Boundary Condition,” International Journal of Emerging Multidisciplinary Fluid Sciences, Vol. 3, , pp. 85-98, 2013.

[9] Selak, R., and Lebon, G., "Bénard-Marangoni Thermoconvective Instability in Presence of a Temperature Dependent Viscosity," Journal De Physique II, Vol. 3, pp. 1185-1199,1993. 
[10] Nield, D. A., "The Effect of Temperature-Dependent Viscosity on the Onset of Convection in a Saturated Porous Medium," Journal of Heat Transfer, Vol. 118, No pp. 803-805,1996.

[11] Straughan, B., "Sharp Global Non-Linear Stability for Temperature Dependent Viscosity," Proceedings of the Royal Society of London, Vol. 458, pp. 1773-1782,2002.

[12] Busse, F. H., and Frick, H., "Square Pattern Convection in Fluids with Strongly Temperature Dependent Viscosity," Journal of Fluid Mechanics, Vol. 150, pp. 451-565, 1985.

[13] V.S. Solomatov, "Scaling of temperature-and-stress-dependent viscosity convection". Phys. Fluids, Vol.7, pp.266-274, 1995.

[14] A. Davaille and P. Jaupart., "Transient high Rayleigh number thermal convection with large viscosity variation". J. Fluid. Mech., Vol. 3, pp.141$166,1993$.

[15] X. Nicolas, "Bibliographic review on the Poiseuillee Rayleigh Bénard flows; the mixed convection flows in horizontal rectangular ducts heated from below", International Journal of Thermal Sciences, vol. 41,pp.135-142, 2002.

[16] Ruan, X. Ye., Chen., Doona, T., Yang, T., "Developments in Ohmic heating, in: P.S. Richardson (Ed.), Improving the Thermal Processing of Foods", Woodhead Publishing in Food Science and Technology, CRC Press, pp.224-252, 2004.

[17] Hill, A. A "Penetrative convection induced by the absorption of radiation with nonlinear internal heat source", Dyn. Atmos. Oceans Vol. 38, pp.57-67, 2004.

[18] Straughan, B., "Stability and wave motion in porous media", Appl. Math. Sci. Ser.vol. 165. Springer, New York, 2008.

[19] Generalis, S. C., Busse, F. H, “Transition in inclined internally heated fluid layers" Proceedings $5^{\text {th }}$ European Thermal-Sciences Conference (1821) 1-8 Eindhoven (NL), 2008.

[20] Nield, D. A., “Throughflow effects on the Rayleigh-Benard convective instability problem”, J. Fluid Mech. Vol. 185,pp. 353-360, 1987.

About the Author
Dr.Gangadharaiah.Y.H, obtained his M Sc from Bangalore University, Bangalore and Ph.D from Visvesvarya
Technological University, Belgaum. He has teaching Engineering Mathematics for undergraduate and post
graduate students for past several years. He has published/ presented more than 30 research papers in national and
international journals/ conferences and his research area of interest are fluid mechanics: convection: stability
analysis.
Mr.Ananda.K, obtained his M.Sc., from Bangalore University, Bangalore and M.Phil., from Sri Venkateswara
University, Tirupati. He has been teaching Engineering Mathematics for undergraduate students for the past 11
years. He has published/ presented more than 10 research papers in national and international journals/
conferences and his research area of interest are fluid mechanics: convection: stability analysis.

